\title{
Desafios para a produção do cuidado na Atenção Primária à Saúde*
}

\author{
Challenges for Primary Health Care Production
}

Retos para la producción asistencial en Atención Primaria de Salud

\section{Camila Tahis dos Santos Silva ${ }^{\mathrm{I}}$, Marluce Maria Araújo Assis ${ }^{\mathrm{II}}$, Mariana Mercês Mesquita Espíndola ${ }^{I I I}$, Maria Angela Alves do Nascimento ${ }^{\text {IV }}$, Adriano Maia dos Santos $\mathrm{V}$}

Resumo: Objetivo: compreender a organização da produção do cuidado em saúde na Atenção Primária. Método: estudo qualitativo, realizado com 23 participantes da equipe de saúde da família por meio de entrevista e observação sistemática em sete Unidades de Saúde da Família da região centro norte da Bahia, entre maio e julho de 2017; análise de dados orientada pela hermenêutica gadameriana. Resultados: a porta de entrada é seletiva; fluxos de atendimento são regulados por oferta focalizada em alguns serviços, com longa espera e dificuldades nos agendamentos de consultas, além de desarticulados e insuficientes para a continuidade do cuidado. Observou-se falta de materiais e condições de trabalho precárias. A avaliação é invisível, com insuficiência de ações que corroborem a satisfação do usuário. Conclusão: evidencia-se necessidade de melhoria na organização do cuidado e composição da Atenção Primária como ordenadora de práticas integrais e resolutivas na rede de atenção.

Descritores: Sistema Único de Saúde; Saúde da Família; Atenção Primária à Saúde; Estratégia Saúde da Família; Serviços de Saúde

Abstract: Objective: to understand the organization of the production of health care in Primary Care. Method: qualitative study, carried out with 23 participants of the family health team by means of interview and systematic observation in seven Family Health Units of the central northern region of

\footnotetext{
${ }^{I}$ Enfermeira, Mestre em Enfermagem, Instituto Federal de Sergipe/IFS, Lagarto, SE, Brasil. E-mail: camila_tahis@hotmail.com, orcid: 0000-0003-2434-2817.

${ }^{\text {II }}$ Enfermeira, Doutora em Enfermagem, Universidade Estadual de Feira de Santana/UEFS, Feira de Santana, BA, Brasil. E-mail: aassis@uefs.br, orcid: 0000-0003-0063-6677.

III Enfermeira, Mestre em Ciências, Instituto Federal de Pernambuco/IFPE, Recife, PE, Brasil. E-mail: marianaespindola@recife.ifpe.edu.br, orcid: 0000-0001-6438-5446.

IV Enfermeira, Doutora em Enfermagem, Universidade Estadual de Feira de Santana/UEFS, Feira de Santana, BA, Brasil. E-mail: angelauefs@yahoo.com.br, orcid: 0000-0002-0616-8133.

V Odontólogo, Doutor em Saúde Pública, Universidade Federal da Bahia/UfBA, Vitória da Conquista, BA, Brasil. E-mail: maiaufba@ufba.br, orcid: 0000-0001-9718-1562.
}

* Extraído da dissertação "Cuidado integral nas dimensões organizativas, técnicas e simbólicas da atenção primária à saúde: narrativas da equipe de saúde e usuários”, Programa de Pós-Graduação do mestrado profissional em enfermagem, Universidade Estadual de Feira de Santana, 2018. 
Bahia, between May and July 2017; data analysis oriented by Gadamerian hermeneutics. Results: the entrance door is selective; service flows are regulated by offering focused on some services, with long waiting and difficulties in scheduling appointments, besides being disjointed and insufficient for continuity of care. A lack of materials and precarious working conditions was observed. The evaluation is invisible, with insufficient actions to corroborate user satisfaction. Conclusion: there is an evident need for improvement in the organization of primary care and composition of primary care as an organizer of integral and resolute practices in the attention network.

Descriptors: Unified Health System; Family Health; Primary Health Care; Family Health Strategy; Health Services

Resumen: Objetivo: comprender la organización de la producción de la atención sanitaria en Atención Primaria. Método: estudio cualitativo, realizado con 23 participantes del equipo de salud de la familia mediante entrevistas y observación sistemática en siete Unidades de Salud de la Familia de la región centro norte de Bahía, entre mayo y julio de 2017; análisis de datos guiado por la hermenéutica gadameriana. Resultados: la puerta de entrada es selectiva; los flujos asistenciales están regulados por una oferta focalizada en algunos servicios, con una larga espera y dificultades para programar citas, además de desarticulados e insuficientes para la continuidad asistencial. Se notó la falta de materiales y malas condiciones de trabajo. La evaluación es invisible, con acciones insuficientes que corroboran con la satisfacción del usuario. Conclusión: existe la necesidad de mejorar la organización de la atención y la composición de la Atención Primaria como organizadora de prácticas integrales y resolutivas en la red asistencial.

Descriptores: Sistema Único de Salud; Salud de la Familia; Atención Primaria de Salud; Estrategia de Salud Familiar; Servicios de Salud

\section{Introdução}

A Atenção Primária à Saúde (APS) considerada a "porta de entrada” do Sistema Único de Saúde (SUS) é o primeiro contato do indivíduo com os serviços de saúde, constituindo-se em alicerce da atenção à saúde. Isso se fundamenta no reordenamento da rede e demais serviços, por meio da coordenação do cuidado, considerando o elo entre as pessoas e suas necessidades de saúde em todos os níveis de complexidade do Sistema. ${ }^{1-2}$

A APS implica maior equidade em suas ações e eficiência nos cuidados de saúde para a continuidade e, consequentemente, menor fragmentação da atenção ao longo da rede, impactando positivamente nos indicadores de saúde. ${ }^{3}$ Por conseguinte, é necessária a compreensão da forma como a APS opera e se relaciona com os demais elementos da rede de atenção, na busca pela produção do cuidado integral. Este é 
compreendido como resultante das ações dos profissionais de saúde, operadas por saberes tecnológicos que valorizem o campo das relações e intersubjetividades que tangem as necessidades dos usuários. ${ }^{1}$ Logo, considerando-a como ordenadora da rede de atenção à saúde, ponderam-se como dimensões analíticas neste estudo: porta de entrada, fluxo de atendimento e avaliação.

O fluxo do atendimento se organiza por meio de diferentes instituições com multiplicidade de agentes e espaços sociais em diversos níveis de densidade tecnológica. Nesse sentido, é parte de uma rede dinâmica e coordenada, que de forma criativa se ajusta às especificidades de cada local ou região. ${ }^{4}$ Os fluxos que correspondem ao itinerário do usuário pela rede, também denominados de referência e contrarreferência, como partes intrínsecas e interconectadas à dinâmica dos processos regulatórios, devem superar o excesso de normas e burocracia e buscar estabelecer elos de significados e sentidos na organização da produção do cuidado. Para tanto, é salutar compor "o acesso, a integralidade e a equidade como organizadores do trabalho em saúde”. 5:4

O dispositivo da avaliação em saúde é fundamental para sistematizar e acompanhar as ações de forma articulada em rede. É um processo que envolve diferentes sujeitos sociais da prática e da comunidade, cujas ações teriam que ser embasadas em planos de intervenção estratégicos e participativos para viabilizar uma atenção responsiva e sistêmica. ${ }^{6}$ Por conseguinte, busca considerar e compreender a satisfação dos sujeitos envolvidos nas práticas de produção de saúde, que resulte em melhoria dos sistemas e resposta às necessidades.

A implementação da APS na forma como foi concebida, com a finalidade de melhorar a qualidade da atenção e diminuir as iniquidades com a oferta de cuidados primários de variada complexidade, requer a superação de várias barreiras, dentre 
elas, as relacionadas à gestão e às redes de cuidado. ${ }^{7-9}$ Uma vez que, resultam na execução de políticas seletivas e correspondem aos entraves no modo de organização das práticas e nas dificuldades relacionadas ao acesso aos serviços no SUS. ${ }^{10-11}$

Outra característica observada em estudos ${ }^{2-3}$ sobre a APS se refere ao modelo de saúde, que valoriza a lógica procedimento-centrada e ações medicalizantes, insuficientes para responder às demandas/necessidades de saúde. Isso remete a indispensabilidade de colocar na agenda de discussão dos profissionais de saúde o modo de organizar e produzir o cuidado, implicando monitoramento e avaliação contínua do processo. ${ }^{6}$

Estudos que discutiram a dinâmica da produção do cuidado na APS na rede SUS, adotaram análises recortadas seletivamente nessas dimensões referidas, mostrando a insuficiente capacidade do primeiro nível de atenção para exercer papel fundamental na ordenação do cuidado em rede, devido à falta de continuidade dos processos de atenção, na composição da referência e contrarreferência. ${ }^{4,9-12}$ As análises dos artigos ${ }^{4,8,13}$ são centradas nos atributos da APS, como longitudinalidade, coordenação, integralidade, elencos de serviços, entre outros, e nas bases organizativas do território, como componente da região de saúde, seus pontos e contrapontos nos sistemas de saúde.

O debate sobre a temática nos instiga relacionar a dimensão organizativa da produção do cuidado na APS, que agrega a porta de entrada, o fluxo de atendimento e a avaliação, como partes de um contexto que se contempla e se complementa na composição observacional e discursiva, mediante as experiências dos sujeitos envolvidos, desvelando o modo como usuários e profissionais de saúde demarcam a organização das práticas. ${ }^{10-14}$ Por conseguinte, emerge a questão de pesquisa: como se organiza a produção do cuidado na APS? O objetivo deste artigo é compreender a organização da produção do cuidado em saúde na Atenção Primária. 


\section{Método}

Estudo qualitativo, orientado pelo referencial da hermenêutica crítica gadameriana para compreensão de narrativas, por meio de entrevista e observação sistemática. Logo, o cerne da teoria de Gadamer é explicar como fugir do círculo fechado das concepções anteriores para manter um constante interpretar, a ponto de substituir os pré-conceitos, por meio da comunicação, por conceitos novos mais adequados aos sujeitos. ${ }^{15}$

A investigação foi realizada em um município localizado na região centro norte do estado da Bahia, Brasil, com população de 75.437 habitantes e 16 equipes de Saúde da Família (EqSF), distribuídas em oito Unidades de Saúde da Família (USF) com uma cobertura populacional de 63,6\%. ${ }^{16}$ O campo empírico contemplou todas as Unidades do município, excluindo-se uma Unidade por não possuir equipe completa no momento da coleta. Logo, foi constituído por sete USF, três localizadas na zona rural e quatro na zona urbana. No processo de seleção das Unidades foram adotados os seguintes critérios: ter no mínimo um ano de funcionamento; atuar com equipe completa ou equipe mínima (médico, enfermeiro, técnico de enfermagem e agente comunitário, podendo ser acrescida de cirurgião-dentista e auxiliar de consultório dentário, como integrantes da equipe multiprofissional). ${ }^{16}$

A coleta foi realizada entre os meses de maio e julho de 2017. A aproximação do campo foi realizada por meio de visitas, resultando numa média de três a quatro visitas por USF, com apresentação do projeto de pesquisa, explicitando objetivos, riscos e benefícios, sigilo, anonimato e privacidade durante a coleta de dados. Os participantes foram selecionados por amostra intencional, de acordo com a disponibilidade manifestada após esse contato inicial. As entrevistas narrativas foram agendadas de acordo com a disponibilidade dos participantes, após o término dos atendimentos. Os critérios de inclusão e exclusão foram determinados segundo os grupos:

- Grupo 1: EqSF/profissionais de saúde, atuantes na USF com no mínimo seis meses de experiência profissional, excluindo aqueles profissionais afastados do serviço, em gozo de licenças médica, 
gestação, prêmio ou afastamento quer para outro serviço, quer para licença sem vencimentos.

- Grupo 2: Usuários do serviço, com idade maior de 18 anos e com cadastro na USF há pelo menos seis meses, excluindo-se os usuários que não faziam uso do serviço há pelo menos um ano.

A quantidade de participantes da pesquisa foi definida com base na repetição exaustiva dos conteúdos presentes nas narrativas, representados no processo de coleta e análise dos dados empíricos, prescindindo de representação de cada categoria por USF $\cdot{ }^{17}$ Compuseram este estudo 23 participantes, sendo 17 profissionais de saúde (grupo 1): seis agentes comunitários de saúde (ACS); seis enfermeiras; duas técnicas de enfermagem; duas médicas; duas odontólogas; e seis usuários dos serviços, prescindindo de representação por USF, tendo em vista a saturação dos dados, bem como a dificuldade no aceite em participar e recusa por um profissional em fazer parte da coleta.

A coleta de dados ocorreu após as visitas às USF. Como instrumento norteador da entrevista e da observação participante foi utilizado um roteiro comum semiestruturado com os seguintes itens: a) identificação numérica do entrevistado e da unidade observada; b) narração e/ou observação das dimensões organizativas da produção do cuidado, referentes à porta de entrada, fluxo de atendimento, regulação (referência e contrarreferência) e avaliação; outras observações ou fala livre do entrevistado, impressão/avaliação do entrevistador sobre a unidade e a narração do entrevistado.

O participante narrou a sua compreensão das referidas dimensões de forma livre as perguntas da pesquisadora entrevistadora, buscando explorar aspectos das dimensões que não foram explanadas pelos entrevistados. Essas foram realizadas in loco, mediante convite e agendamento prévios no momento das visitas, geralmente ao final dos atendimentos, em sala reservada para este fim. Com média de 15 minutos por participante, totalizando aproximadamente seis horas, sendo áudio-gravadas, transcritas, interpretadas e analisadas. As observações participantes ocorreram concomitante às entrevistas e tiveram a duração de 168 horas, em média 24 horas por USF abrangendo os dois turnos de atendimento (manhã e tarde) e registradas no diário de campo. Os aspectos observados relacionados à dimensão organizativa 
foram porta de entrada, barreiras geográficas, regulação-fluxo e contra fluxo, e avaliação.

A observação participante compôs a análise das narrativas, pois possibilitou o entrecruzamento das informações, apreendida com o conhecimento acerca das experiências vividas pelas pessoas associadas aos significados, atividades ou eventos, edificando os aprendizados presentes entre as representações, atividades ou eventos, experiências vividas e a observação da realidade. ${ }^{18-20}$ Tal análise foi dividida em etapas para melhor compreensão e interpretação, a saber:

Etapa 1 - Fase de distanciamento: consistiu na transformação da língua falada em língua escrita, na transcrição das entrevistas e registro das observações em texto.

Etapa 2 - Fase de apropriação: envolveu as leituras e entendimento geral dos textos para apropriação do seu significado, transformando-o em algo familiar, próprio, segundo suas mediações de conhecimentos, experiências e realidade observada.

Etapa 3 - Fase de explicação e organização dos dados: submeteu-se os textos à análise do software NVivo, versão 11 Pro for Windows, para organização dos dados em unidades de análises, que emergiram das convergências das falas e das observações. Tal estratégia buscou proporcionar coerência na interpretação dos dados com o armazenamento e a descrição de forma sistematizada das unidades de análise para seguimento dos demais passos inerentes à interpretação, compreensão e construção das representações dos grupos e do que foi observado.

Etapa 4 - Fase de compreensão: ocorreu um processo interativo que possibilitou às pesquisadoras-leitoras, uma compreensão ampla do que foi dito e observado na realidade dos serviços, e que depende dos conhecimentos prévios do leitor para seu desvelar. ${ }^{18}$

Ressalta-se que esta pesquisa obteve aprovação do Comitê de Ética e Pesquisa da Universidade Estadual de Feira de Santana em 29 de abril de 2017, sob o número do protocolo 2.039.015, em seguimento às normas da Resolução 466/2012 do Conselho Nacional de Saúde.

A identificação das narrativas é representada de acordo com cada grupo de participantes deste estudo. Os nomes citados após cada narrativa foram codificados para resguardar o 
anonimato, tendo os profissionais sido identificados pela inicial E (de Equipe), seguida de um número, e os usuários identificados pela inicial U (de Usuário), seguida de um número, de acordo com a ordem crescente da realização de cada entrevista.

\section{Resultados}

As sínteses dos resultados das observações são representadas nas Figuras 1 e 2, complementadas pelas narrativas dos participantes (profissionais de saúde e usuários) que operam no cotidiano da APS, no espaço da USF. Logo, foram recortadas as dimensões organizativas da produção do cuidado, desde a porta de entrada até a regulação e avaliação do processo. Como ponto de partida, demarca-se o fluxo organizativo da organização do cuidado na APS como integrante de uma rede de atenção, estabelecida na composição da referência e contrarreferência que é instituída pela regulação para os serviços de média e alta complexidade (Figura 1).

Figura 1 - Representação do fluxo organizativo da produção do cuidado na Atenção Primária a Saúde em um município baiano. Bahia (BA), Brasil, 2017. 


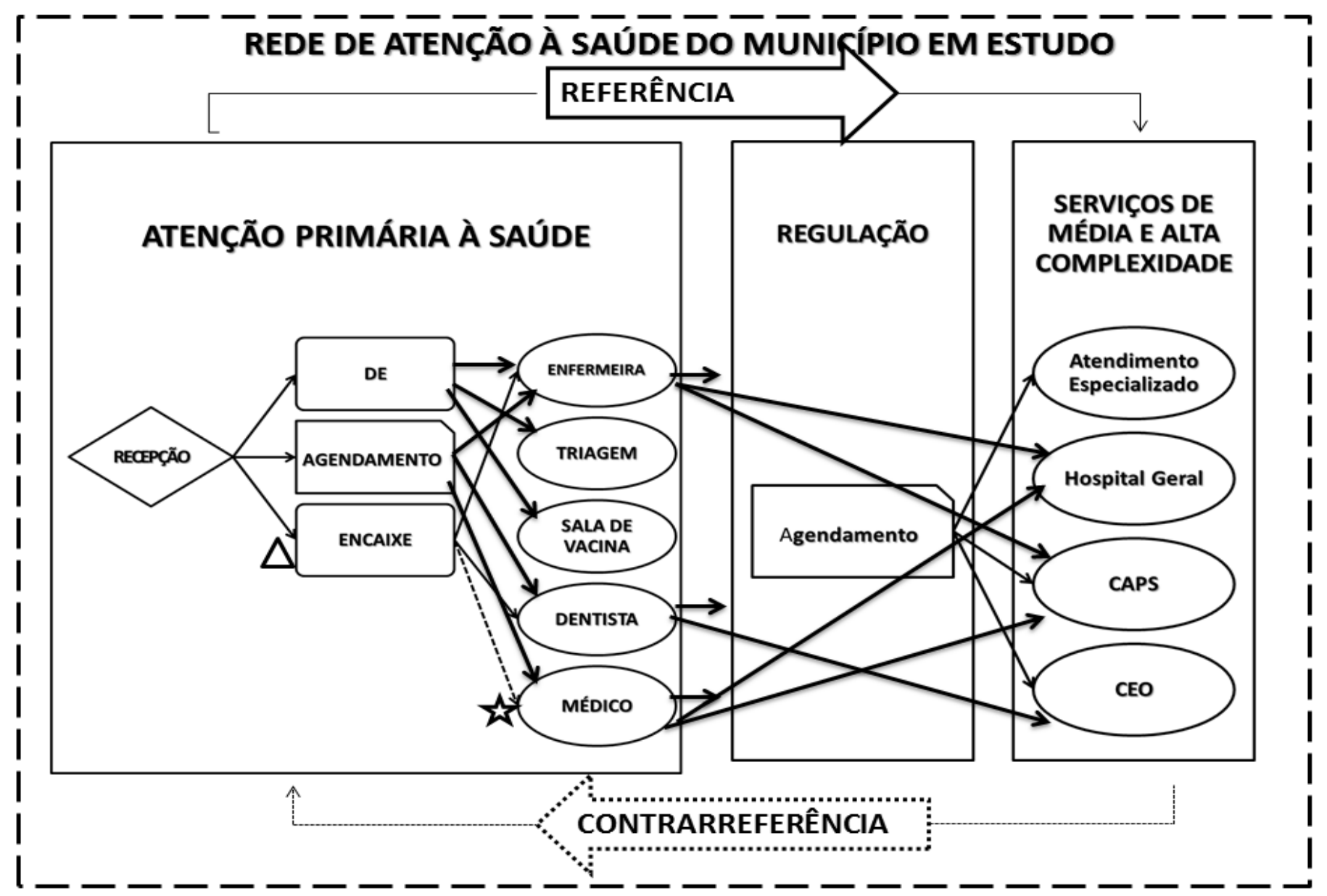

\section{LEGENDA}

DE - Demanda Espontânea

CAPS • Centro de Atenção Psicossocial

CEO - Centro de Especialidades Odontológicas

- Elementos da Rede de Atenção à Saúde

$\square$ - Níveis de complexidade

- Etapa decisória de organização do fluxo de acesso aos serviços da APS

- Forma alternativa de acesso

- Forma de acesso convencional com demora/atraso
- Serviços oferecidos

$\longrightarrow$ - Fluxo de encaminhamento

$-\rightarrow \quad$ Fluxo de encaminhamento com fragilidade

• Serviço mais destacado/demandado

$\bigwedge$ - Forma negociada de acesso sem garantia

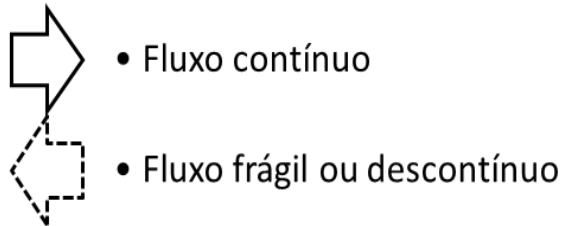


Observa-se na Figura 1, as formas de acesso e entrada na APS, tendo como espaço de intervenção a USF, por meio da recepção que designa e organiza a "porta de entrada" da rede SUS. O fluxo de atendimento é ordenado por meio de agendamentos com base em ações programáticas, já a demanda espontânea (DE) ou de “encaixe”, caracteriza-se pelas linhas pontilhadas, por ser considerada uma das fragilidades da organização do fluxo. A DE consiste numa forma de acesso alternativa e negociada entre os sujeitos para acessar as consultas médicas, caso tenha alguma necessidade persistente ou outras situações que facilitem sua entrada no serviço de saúde. As narrativas retratam como se operacionaliza o acesso na "porta de entrada” do sistema de saúde.

Só assim, na hora de marcar que demora um pouco. A marcação demora, às vezes a gente vem e não tem vaga [...] Só essa questão mesmo na hora da marcação que é difícil demais [...]. (U1)

Meu marido trabalha na saúde, então é tudo facilitado. Tem que marcar as consultas na casa de marcação, depois eu venho para ser atendida. E é assim que acontece. Tem que ter paciência para aguardar a sua vez. [...] Eu cheguei lá e estava precisando, foi uma situação em que não tinha mais vaga, mas a atendente foi lá e falou com o profissional e ele cedeu a vaga. (U5)

Quando chego aqui, o pessoal da recepção, só vai marcar 11 horas [...] E o paciente que chegou às 8 h, vai fazer como? Vai voltar para vir às 11 horas? (E04)

Procuro os agentes comunitários para saber se tem médico, pergunto: " $A$ h, quem está consultando lá?" Porque também, eles faltam bastante. [...] Venho para a doutora sempre, meu prontuário é com ela. [...] As agentes comunitárias sempre informam bem, o dia que tem médico, o dia que estão fazendo exame, o dia que estão marcando, sempre, e tem duas agentes comunitárias, que são vizinhas, que sempre informam bem.(U6) 
A pessoa está precisando da consulta, vem aqui e, às vezes, é mal recebido. Já aconteceu até de dizer que não tem vaga, e outras pessoas conseguirem [...]. A gente vai para um lugar, a gente vai querer ser bem acolhido e não é. (E3)

A compreensão dos usuários é destacada por meio das dificuldades no acesso desde a "porta de entrada" que seria essencial para a continuidade do processo terapêutico, assinalando entraves nas marcações e agendamentos de consultas. Essa situação tem reflexos na insatisfação e nas formas de enfrentamento do problema, tais como aguardar o agendamento, ou a inclusão do usuário na atenção à demanda espontânea, por meio da negociação e diálogo com os profissionais de saúde. A Figura 2 evidencia estas características.

Figura 2 - Dimensões organizativas na Atenção Primária à Saúde de um município baiano. Bahia (BA), Brasil, 2017.

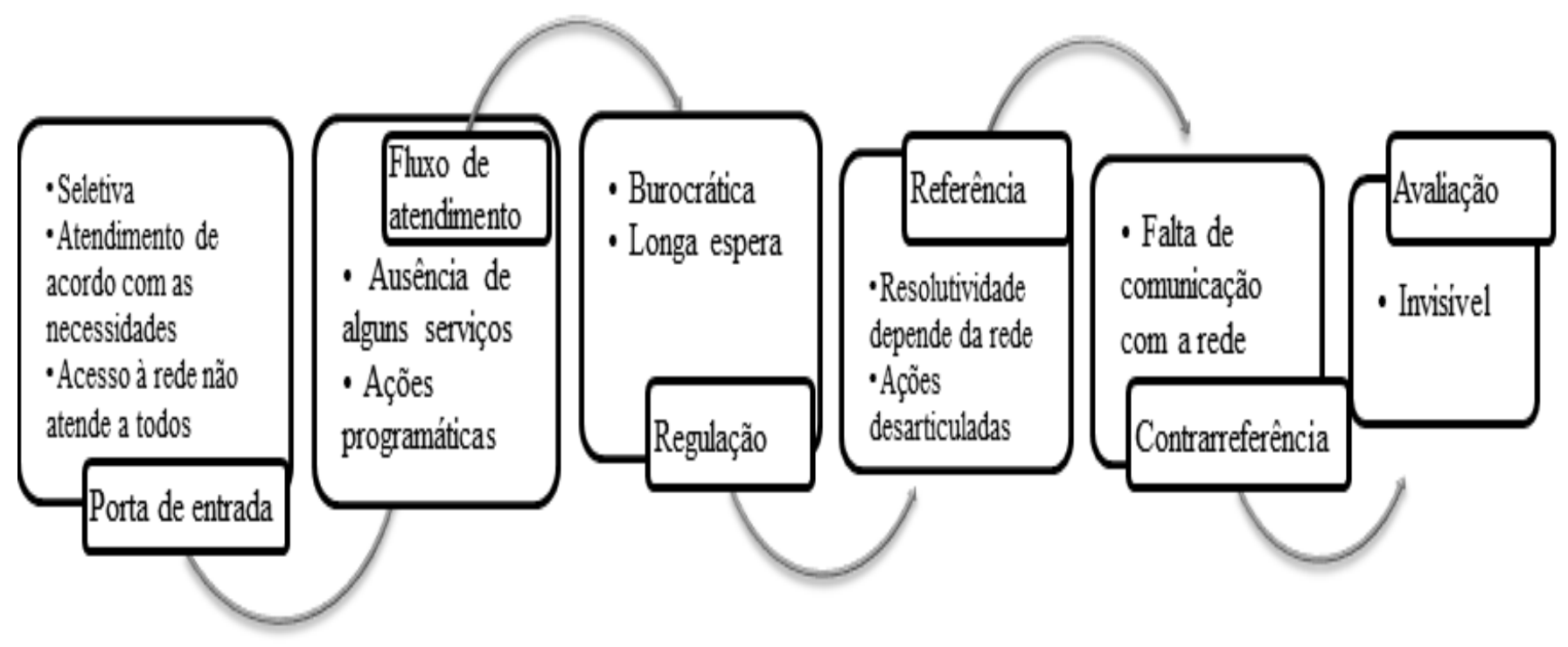

A Figura 2 mostra que a "porta de entrada" é seletiva e organizada de acordo com as necessidades focalizadas em demandas pontuais, como agravos específicos relacionados as ações programáticas, a exemplo do Programa de Hipertensão, Imunização, entre outros. O fluxo de atendimento é regulado por meio da oferta de serviços tais como: consultas de enfermagem, 
consultas médicas, triagem, sala de vacina, atendimento odontológico e assistência farmacêutica. Em algumas unidades observou-se oferta de serviços como consultas de especialistas. Particularmente, as unidades da zona rural não dispõem do profissional médico na equipe, ou este atende apenas esporadicamente em dias alternados da semana. Atentou-se também que, em outras unidades, o fluxo é prejudicado pela insuficiência de materiais, equipamentos e sua manutenção, como demonstrado nas sínteses das narrativas:

Esses dias, atualmente, a cadeira também está com problema e o dentista não está atendendo. (E15)

Pois, se você não tem uma sala bem equipada, se falta maca para atendimento de uma urgência, para aquele paciente que demanda um cuidado maior e não tem uma maca, não tem um ar-condicionado na sala para você fazer uma consulta, de forma que o ambiente esteja mais receptivo e agradável àquele usuário. (E9)

Quando eu venho, pego remédio quando não tem, eu pego lá na rua [em outra USF]. (U2)

Entrei em contato com a enfermeira, que mesmo sem o carro para levar na localidade distante em que a senhora morava, se disponibilizou a ir fazer esse curativo, a pé mesmo. (E10)

Com relação ao fluxo de atendimento observado (Figura 1 e 2), a referência acontece pelo encaminhamento do enfermeiro, médico ou dentista que direcionam o usuário aos demais pontos da rede de atenção, de acordo com as demandas que se apresentam na unidade. Outrossim, a regulação para acesso a outros serviços ocorre por meio de agendamentos na central de marcação, ou na própria unidade, viabilizado por sistema de cotas, nos casos de unidades da zona rural. Os recortes de falas elucidam tais percepções:

[...] paciente que precisa de uma especialidade com urgência, ou a médica encaminha, eu já tento marcar daqui mesmo com a central de marcação. 
Mas, quando é um paciente que dá para gente esperar, aí a gente encaminha, por solicitação, e ele vai para central de marcação, marca essa consulta e faz o atendimento. (E14)

Ele [o paciente] traz a solicitação dos exames, a solicitação de especialistas. Ele vem aqui, a gente tem a cota, com a quantidade semanal para isso, e toda segunda-feira o motorista leva à central de marcação, para que os pacientes não precisem ir até cidade marcar. (E18)

Quanto às referências dos casos de urgência, estas prescindem de marcação e são realizadas diretamente no hospital ou centro de especialidades odontológicas, assim como os casos que necessitam dos serviços do Centro de Atenção Psicossocial (CAPS) que também abstraem de marcação Centro de Atenção Psicossocial I, que tem como foco usuários adultos com transtornos mentais graves e persistentes (CAPS-I) e Centro de Atenção PsicossocialÁlcool e Drogas (CAPS -AD). A contrarreferência é representada na Figura 1 por linhas pontilhadas, caracteriza-se como fragilidade, uma vez que, em muitos casos não ocorre o retorno do processo de atenção, referido pelo próprio usuário e/ou pelos agentes comunitários de saúde (ACS).

Se eu mandar o paciente para referência, na contrarreferência os prontuários não são compartilhados, e a ficha de contrarreferência não manda para a equipe. (E5)

[...] dificilmente há retorno. Não há retorno dos próprios profissionais em estar contrarreferenciando aquele paciente para que aquele cuidado se dê de forma contínua. (E9)

No verso dela [ficha] é para ser feita a contrarreferência e essas fichas nunca voltaram. [...] encaminha para rede e eles [profissionais de saúde] não dão retorno. Aí a gente não tem como fechar um caso. (E13)

Ademais, quanto à avaliação dos serviços (Figura 2), esta não é sistematizada e nem estabelecida de forma contínua, cuja expressão torna-se invisível nos serviços, o que se 
configura como um descompasso para a continuidade e coordenação do cuidado. Os dados empíricos evidenciam, portanto, um fluxo organizativo da produção do cuidado na APS fragmentado e em desacordo com práticas integrais e que respondam de fato às necessidades daqueles que demandam a atenção.

A última coisa que eu fiz aqui, e não deu certo. Não deu certo, assim, foi um preventivo que eu fiz e não veio o resultado. (U3)

Sinto-me cuidada, um pouco. Porque meus exames eu não faço como o médico pede. Porque não tem como marcar. Não tem vaga. Mas fora isso, me sinto um pouco cuidada. (U5)

Além disso, ressalta-se o protagonismo da enfermeira, destacada nas narrativas a seguir, enquanto organizadora da produção do cuidado, com responsabilização em vários serviços, desde a definição e organização de cronograma até o acesso dos usuários. As intervenções são baseadas em ações programáticas, práticas educativas e coordenação da USF.

[...] a enfermeira que coordena o atendimento, os profissionais, a unidade em si. (E12)

[...] quem coordena, na verdade, são as duas enfermeiras. (E14)

[...] a maioria das atividades quem acaba tomando partido, no caso, posso falar que sou eu, se é uma programação de uma atividade educativa, se é a [pausa] realização do cronograma, da unidade, se é para fazer, para ajudar no programa de saúde nas escolas, enfim, todas as atividades quem acaba tomando iniciativa é o enfermeiro. (E18)

Os resultados das narrativas e figuras se complementam na evidenciação dos elementos organizativos captados pelos instrumentos possibilitando a triangulação que será discutida adiante.

\section{Discussão}


15 | Silva CTS, Assis MMA, Espíndola MMM, Nascimento MAAN, Santos AM

A APS como "porta de entrada” da rede SUS engloba as características locorregionais e leva em consideração o contexto da comunidade. A aproximação dos sujeitos estreita as relações e torna todo o ambiente fértil às relações de cuidado, constituindo a Saúde da Família como espaço social em que deveria se concretizar a entrada do usuário no sistema de saúde. ${ }^{8}$

Os dados empíricos evidenciam barreiras, como dificuldades no acesso a consultas, barreiras geográficas relacionadas às unidades da zona rural, morosidade no acesso dos usuários que enrijecem as relações de cuidado e resultam em experiências assistenciais frágeis e com baixa ou insuficiente resolubilidade. O acesso na "porta de entrada" que seria essencial ao desencadeamento do cuidado progressivo em rede sofre o impacto das dificuldades organizativas que se refletem nas barreiras enfrentadas no cotidiano da APS e que trazem características que limitam o acesso a este nível de atenção, fruto da ineficiência e atenção fragmentada. Assim, a APS apesar de oportunizar uma aproximação inicial entre os sujeitos e a rede de saúde enfrenta dificuldades no acesso e vínculo que são considerados essenciais para a conformação de cuidado responsivo e integral.

O ACS, no decorrer da sua prática pode contribuir para a melhoria do acesso e do fluxo do atendimento, sendo um elo com a comunidade. Ele pode facilitar o acesso na "porta de entrada" por meio do trabalho de reconhecimento da sua área de atuação e demarcação territorial, fortalecendo os vínculos com os usuários e integrando demandas/necessidades com a equipe de saúde da família. O ACS desenvolve o papel de apropriação e pertencimento, desencadeando tais significados nos usuários que podem compartilhar suas experiências, necessidades e tradições em comum..$^{21}$

Neste estudo, a orientação do fluxo acontece conforme o modelo de medicalização da saúde, centrado na doença, com foco na queixa para conduta profissional, tendo como aspectos limitantes a seletividade no primeiro contato e entre os níveis de atenção; e a organização do atendimento pautada em ações programáticas ou pela priorização de agravos específicos. 
Estudo realizado na APS, acerca da orientação de saúde da criança no Brasil, identificou a permanência de características do modelo médico-hegemônico, que desencadeia limitações aos princípios centrais da atenção básica como parte de uma política pública, que ainda não têm a família como foco da atenção, predominando as ações curativas em saúde. ${ }^{9}$ Além disso, apreendeu-se na realidade experienciada que dificuldades no fluxo dos usuários pode resultar em fragilidades na resolubilidade dos atendimentos, debilidades no referenciamento para a rede de atenção e descontinuidade do cuidado e prestação de assistência.

Ante o exposto, a organização do fluxo de atendimento ocorre de forma limitada, como foi destacado nas observações (Figura 1 e 2) e nas narrativas dos participantes. Situações que, muitas vezes, se iniciam no momento em que esse usuário chega à unidade, seja para a procura do serviço ou por intermédio dos ACS. Esse fato leva a segmentação do atendimento e à sua insatisfação em busca de atendimento às suas necessidades.

Portanto, é necessário o fortalecimento no modo de organização do cuidado por meio de iniciativas que propiciem ampliar a oferta de vagas para atendimento, fornecimento de insumos e materiais e melhorias na infraestrutura por meio do aumento do financiamento e dos recursos para APS. Além da capacitação e fortalecimento de vínculo das equipes, provedoras dos cuidados primários.

Ante o exposto, considera-se que as dificuldades enfrentadas dentro das unidades levam ao descrédito dos serviços ofertados na APS. Corroborando resultados similares a outros estudos em que os usuários duvidaram do poder de resolubilidade que esse nível de atenção dispõe. ${ }^{1,12}$ Outras dificuldades consideradas expressivas se referem à falta de materiais e às condições precárias de trabalho nas USF, conforme as narrativas dos entrevistados, comprometendo muitas vezes as práticas e o serviço, tornando-os limitados e pouco resolutivos, contribuindo com o descontentamento dos profissionais e insatisfação dos usuários.

Achado semelhante foi encontrado no estudo, ${ }^{9}$ tendo apresentado os problemas 
estruturais de inadequação de espaço físico com caráter de improvisação, condições de trabalho impróprias para atendimento assistencial e a escassez de equipamentos e instrumentos fundamentais de trabalho como entrave para produção do cuidado e resolubilidade da APS, levando a descrédito dos usuários no serviço.

Outrossim, a inexistência do médico em algumas unidades, principalmente na zona rural, corrobora a ausência de serviços essenciais na saúde da família, representando um comprometimento no acesso, no atendimento, na qualidade da atenção e em práticas integrais. ${ }^{1,}$ 9-12 Estes estudos corroboram a realidade encontrada ao adentrar nas USF da zona rural em que há grande rotatividade de profissionais, em especial médicos, com pouca vinculação dos profissionais, e consequentemente, práticas superficiais e incipientes.

Com relação ao fluxo de atendimento, ${ }^{22-23}$ este se configura em descompasso para a continuidade do cuidado, entendido como essencial para a manutenção da produção do cuidado de forma coordenada e perene, mesmo que por diferentes profissionais ou níveis de complexidade, quer seja pela construção de práticas e saberes isolados, ou pela manutenção de uma rede que pouco se comunica, descaracterizando a APS como coordenadora da rede SUS. Os resultados do presente estudo vão ao encontro destes achados, visto que o referenciamento de usuários era regulado de acordo com as necessidades de cada um, que a depender são encaminhados para diferentes serviços como CAPS, médicos especialistas ou serviços hospitalares.

Constatou-se, no decorrer do processo da coleta de dados que, nem sempre o encaminhamento do usuário a outro serviço atendia às suas necessidades pela falta de vagas, práticas isoladas e sem interação de profissionais de saúde no trabalho em equipe para a produção do cuidado, mantendo o foco da atenção apenas na demanda que originou o encaminhamento.

Diante do exposto, tais dificuldades repercutem na dinâmica do cuidado prestado ao usuário, com fragmentação do processo terapêutico no percurso da rede SUS. Nesse sentido, a referência e a contrarreferência, que são dispositivos essenciais para o cuidado e para a consolidação de uma APS 
abrangente, mostram-se frágeis, descontínuas e com insuficiente resolubilidade.

Estudos $^{11,22}$ semelhantes corroboram esses descompassos, incluindo o trabalho da equipe saúde da família incompleta, a falta de reconhecimento sobre o funcionamento da rede e a deficiência de articulação e comunicação entre os níveis de atenção. Esse trabalho é fragmentado, traduzindo-se na ausência de retorno dos serviços para os quais o usuário foi referenciado e com a baixa responsabilização de alguns integrantes da equipe envolvidos no cuidado, que muitas vezes, comprometem a efetividade dos sistemas de referência e contrarreferência. Tais sistemas encontram resistência e impasses para a sua efetivação, sendo necessária a sensibilização e educação permanente em saúde aos profissionais acerca da rede de atenção, com vistas a oferecer ao usuário um cuidado integrado, multidisciplinar e interdisciplinar. ${ }^{22}$

Os achados traduzem como alternativa para essa limitação de cooperação/comunicação entre os profissionais e níveis de atenção, que a equipe busca essas informações com o próprio usuário, em diferentes momentos da produção do cuidado: nas consultas de retorno com o médico, no atendimento do enfermeiro e na interlocução com o ACS, que coleta a informação no próprio domicílio, assim que os usuários retornam dos serviços aos quais foram referenciados. É o que corrobora demais estudos publicados. ${ }^{11,22-24}$

Embora o cuidado seja reconhecido como uma prática que pode ser realizada por todos os profissionais de saúde e construído entre os níveis de atenção, principalmente na "porta de entrada” do SUS, na prática não foi observado um trabalho em equipe. Tais dificuldades repercutem na forma como os profissionais realizam o cuidado construído na rede, limite à realização da avaliação, elemento crucial que compõe a dimensão organizativa. ${ }^{6}$ Depreende-se pelos dados empíricos analisados que a avaliação pode ser considerada como invisível nos serviços, pois, apesar de requerer mecanismos institucionais que a realizem, aparentemente, deixa a impressão de que ocorre sem sistematização, ou mesmo critérios transparentes por parte da unidade de saúde.

Como dispositivo essencial para sistematização e acompanhamento de ações de forma 
articulada em rede, nota-se como esses descompassos na avaliação implicam desde a descontinuidade do cuidado, pois da mesma forma que requer o envolvimento de diferentes sujeitos, sejam profissionais e usuários implicam também na realização de ações embasadas nos planos de intervenção de forma estratégica e participativa. ${ }^{6}$ Assim, a ausência ou incipiência na sua realização repercutem no pouco conhecimento sobre a satisfação dos usuários, bem como na proposição de melhorias nas práticas de saúde que responda às reais necessidades.

Ademais, destaca-se a atuação do enfermeiro, enquanto organizador da unidade e com uma prática comprometida e responsiva em vários serviços da APS, envolvendo a prestação do cuidado, de modo a elucidar sua relevância no processo terapêutico e de gestão da unidade. Estudo evidencia o protagonismo desse profissional como articulador do processo organizacional, atuando em várias frentes, enquanto líderes das equipes de saúde da família. ${ }^{21} \mathrm{~A}$ liderança do enfermeiro é considerada salutar no fortalecimento dos vínculos, na resolução de conflitos e na condução das situações do cotidiano assistencial, influenciando diretamente no processo de trabalho em saúde.

As limitações deste estudo situam-se na definição de apenas dois segmentos de participantes (profissionais de saúde e usuários) a partir das USF de um município. Em contrapartida, os achados poderão embasar estudos posteriores que busquem a compreensão da dimensão organizativa a partir das narrativas dos demais sujeitos, incluindo os gestores do SUS, outros municípios e demais componentes da rede de atenção à saúde.

\section{Conclusão}

Os resultados do estudo sinalizam que a APS, ainda não se constituiu em "porta de entrada" da rede SUS, sendo seletiva e organizada de acordo com demandas pontuais e agravos específicos. O fluxo de atendimento é ordenado por meio de agendamentos com base em ações programáticas e a demanda espontânea é uma alternativa negociada entre os sujeitos para acessar as consultas médicas. Os processos de avaliação da organização do cuidado são assistemáticos e invisíveis em 
descompasso com a continuidade do atendimento e de práticas coordenadas e integrais.

De acordo com a realidade analisada foi possível compreender como a APS oorganiza-se no espaço de intervenção da saúde da família, assim como a importância de ressignificar a organização da produção do cuidado, ultrapassando uma atenção seletiva para práticas integrais com responsabilização dos profissionais em diferentes níveis de complexidade do sistema.

Todavia esforços precisam ser provocados e assumidos para gerarem mudanças necessárias para o estabelecimento e à concretização da ESF como forma preferencial de organização da APS, mediante as melhorias nos níveis de saúde da população para que sejam compartilhadas e divulgadas por estudos com foco em práticas criativas e inovadoras para transformar fragilidades em potencialidades.

O estudo contribuiu para o reconhecimento da forma como a organização da produção do cuidado é compreendida e realizada, bem como colabora para o fortalecimento da organização das práticas na atenção primária à saúde da identificação de fragilidades que necessitam de atenção e comprometimento por parte dos profissionais de saúde. Destaca-se a atuação dos enfermeiros que tem exercido protagonismo na articulação e coordenação das atividades nas unidades de saúde da família, situação identificada nos dados empíricos, favorecendo o fortalecimento da profissão, enquanto prestadora de uma atenção comprometida e responsável.

\section{Referências}

1. Assis MMA, Nascimento MAA, Pereira MJB, Cerqueira EM. Comprehensive health care: dilemmas and challenges in nursing. Rev Bras Enferm. 2015:68(2):333-8. doi: https://doi.org/10.1590/00347167.2015680221i

2. Freitas MAS, Araújo MRN. As Redes de Atenção à Saúde nos 30 anos do Sistema Único de Saúde: histórias, propostas e desafios. Rev Bras Polít Públicas. 2018:8(3):14-33. doi: https://doi.org/10.5102/rbpp.v8i3.5739

3. O’Malley A, Rich E. Measuring comprehensiveness of primary care: challenges and opportunities. J Gen Intern Med. 2015:30(Sup 3):568-75. doi: https://doi.org/10.1007/s11606-015-3300-z 
4. Almeida PF, Medina MG, Fausto MCR, Giovanella L, Bousquat A, Mendonça MHM. Coordenação do cuidado e Atenção Primária à Saúde no Sistema Único de Saúde. Saúde Debate. 2018:42(1):244-60. doi: https://doi.org/10.1590/0103-11042018S116

5. Freire MP, Louvison M, Feuerwerker LC, Chioro A. Bertussi D. Regulação do cuidado em redes de atenção: importância de novos arranjos tecnológicos. Saúde Soc. 2020:29(3):ei90682. doi: https://doi.org/10.1590/s0104-12902020190682

6. Ribeiro LA, Scatena JH. A avaliação da atenção primária à saúde no contexto brasileiro: uma análise da produção científica entre 2007 e 2017. Saúde Soc. 2019:28(2):95-110. doi: https://doi.org/10.1590/s010412902019180884

7. Ministério da Saúde (BR). Atenção Primária e Promoção da Saúde [Internet]. Brasília (DF): Conselho Nacional de Secretários de Saúde (CONASS); 2011 [acesso em 2017 fev 02]. Disponível em: http://www.conass.org.br/bibliotecav3/pdfs/colecao2011/livro_3.pdf

8. Santos L. Healthcare regions and their care networks: an organizational-systemic model for SUS. Ciênc Saúde Colet. 2017:22(4):1281-9. doi: http://dx.doi.org/10.1590/1413-81232017224.26392016

9. Damasceno SS, Nóbrega VM, Coutinho SED, Reichert APS, Toso BRGO, Collet N. Saúde da criança no Brasil: orientação da rede básica à Atenção Primária à Saúde. Ciênc Saúde Colet. 2016:21(9):2961-73. doi: https://doi.org/10.1590/1413-81232015219.25002015

10. Assis MMA. Redes de atenção à saúde e os desafios da atenção primária à saúde: um olhar sobre o cenário da Bahia. In: Almeida PF, Santos AM, Souza MKB, organizadores. Atenção primária à saúde na coordenação do cuidado em regiões de saúde. Salvador: EdUFBA; 2015. p. 45-64.

11 Hirdes A. A perspectiva dos profissionais da Atenção Primária à Saúde sobre o apoio matricial em saúde mental. Ciênc Saúde Colet. 2015;20(2):371-82. doi: https://doi.org/10.1590/141381232015202.11122014

12. Giovanella L. Atenção básica ou atenção primária à saúde? Cad Saúde Pública. 2018:34(8):1-5. doi: https://doi.org/10.1590/0102-311X00029818

13. Baum F, Freeman T, Sanders D, Labonté R, Lawless A, Javanparast S. Comprehensive primary health care under neo-liberalism in Australia. Soc Sci Med. 2016;168(1):43-52. doi: https://doi.org/10.1016/j.socscimed.2016.09.005

14. Cavalcanti RP, Cruz DF, Padilha WWN. Desafios da regulação assistencial na organização do Sistema Único de Saúde. Rev Bras Ciênc Saúde. 2018:22(2):181-8. doi: https://doi.org/10.4034/RBCS.2018.22.02.12

15. Gadamer HG. Verdade e método I - traços fundamentais de uma hermenêutica filosófica. 15ª ed. Rio de Janeiro: Vozes; 2015. 
16. Departamento de Atenção Básica - DAB [Internet]. Brasília (DF): Ministério da Saúde; 2015 [acesso em 2017 mar 12]. Disponível em: http://dab.saude.gov.br/historico_cobertura_sf/historico_cobertura_sf_relatorio.php

17. Minayo MCS. Amostragem e saturação em pesquisa qualitativa: consensos e controvérsias Rev Pesqui Qual [Internet]. 2017 [acesso em 2020 out 14];5(7):01-12. Disponível em: https://edisciplinas.usp.br/pluginfile.php/4111455/mod_resource/content/1/Minayosaturacao.pdf

18. Ricoeur P. Hermeneutics and the human sciences. Cambridge: Cambridge University Press; 1995.

19. Good B. Medicine, rationality, and experience. Cambridge: Cambridge University Press; 1994.

20. Geanellos R. Exploring Ricoeur's hermeneutic theory of interpretation as a method of analyzing research texts. Nurs Inq. 2016;7(1):112-9. doi: https://doi.org/10.1046/j.1440-1800.2000.00062.x

21 Silva SS, Assis MMA. Family health nursing care: weaknesses and strengths in the Unified Health System. Rev Esc Enferm USP. 2015;49(4):603-9. doi: https://doi.org/10.1590/S0080-623420150000400010

22. Brondani JE, Leal FZ, Potter C, Silva RM, Noal HC, Perrando MS. Desafios da referência e contrarreferência na atenção em saúde na perspectiva dos trabalhadores. Cogitare Enferm. 2016: 21(1):018 doi: https://doi.org/10.5380/ce.v21i1.43350

23. Viacava F, Bellido JG. Health, access to services and sources of payment, according to household surveys. Ciênc Saúde Colet. 2016;21(2):351-70. doi: https://doi.org/10.1590/1413-81232015212.19422015

24. Pinheiro FMFM, Santos OC, Silva JCB, Barbosa LAL, Carvalho LV, Mota SMA. The leadership nurses profile in primary health care. Rev Eletrônica Acervo Saúde. 2020;43:1-7. doi: https://doi.org/10.25248/reas.e2793.2020

Editora Científica Chefe: Cristiane Cardoso de Paula

Editora Associada: Carine Vendruscolo

\section{Autor correspondente}

Mariana Mercês Mesquita Espíndola

E-mail: marianalb13@hotmail.com

Endereço: Av. Prof. Luís Freire, 500 - Cidade Universitária, Recife - PE.

CEP: $50740-545$ 


\section{Contribuições de Autoria}

\section{1 - Camila Tahis dos Santos Silva}

Concepção ou desenho do estudo/pesquisa, análise e/ou interpretação dos dados, revisão final com participação crítica e intelectual no manuscrito.

\section{2 - Marluce Maria Araújo Assis}

Concepção ou desenho do estudo/pesquisa, análise e/ou interpretação dos dados, revisão final com participação crítica e intelectual no manuscrito.

\section{3 - Mariana Mercês Mesquita Espíndola}

Análise e/ou interpretação dos dados, revisão final com participação crítica e intelectual no manuscrito.

\section{4 - Maria Angela Alves do Nascimento}

Concepção ou desenho do estudo/pesquisa, análise e/ou interpretação dos dados, revisão final com participação crítica e intelectual no manuscrito.

\section{5 - Adriano Maia dos Santos}

Análise e/ou interpretação dos dados, revisão final com participação crítica e intelectual no manuscrito.

\section{Como citar este artigo}

Silva CTS, Assis MMA, Espíndola MMM, Nascimento MAAN, Santos AM. Desafios para a produção do cuidado na Atenção Primária à Saúde. Rev. Enferm. UFSM. 2021 [Acesso em: Anos Mês Dia]; vol.11 e30: 1-22. DOI: https://doi.org/10.5902/2179769246850 